\title{
Modelling and Simultaneous Estimation of State and Parameters of Traffic System
}

\author{
Pavla Pecherková1, Jindřich Duník² and Miroslav Flídr² \\ ${ }^{1}$ Research Centre Data-Algorithms-Decision Making, \\ Dept. of Adaptive Systems, Institute of Information Theory and Automation, AV ČR, \\ Pod vodárenskou věži 4, 18208 Prague 8, \\ ${ }^{2}$ Research Centre Data-Algorithms-Decision Making, \\ Faculty of Applied Sciences, University of West Bohemia, \\ Univerzitní 8, 30614 Pilsen, \\ Czech Republic
}

\section{Introduction}

Majority of large cities suffers from traffic congestion, which results in both higher economic costs (caused by larger travel times, increased fuel consumption and more traffic accidents) and degradation of the environment (air pollution, noise, injuries). The traffic congestion forms because of insatisfactory capacity of traffic network or its insufficient exploitation.

The capacity of traffic network can be increased in several ways. These ways can be divided into three groups: (i) construction of new roads, (ii) additional traffic restrictions and (iii) improved traffic control. The first way, i.e. construction of new roads, lanes and intersections, is very complicated and expensive. The capacity is increased but at the price of vast investment costs. In historical centres of cities, constructions of new roads is even impossible because of protected historical areas. The second way is based on the decreasing number of vehicles in the traffic network by restrictions, e.g. decreasing number of entry and exit points of the traffic network. This measure is, however, highly unpopular among the drivers. The last way is deployment of a suitable traffic control system.

The main aim of traffic control is not to increase the capacity of whole traffic network but to increase troughput and thus to alleviatte the influence of bottlenecks. Bottleneck is a place (road, lane, intersection) in traffic network where the capacity is much smaller than capacity of the neighboring roads. In urban traffic networks the intersections, accidents, and closures are the main bottlenecks. The important intersections are equipped with traffic lights which can influence the behaviour of traffic flow. Thus, increasing traffic network capacity by traffic lights control is one possible way for cities with historical centres, such as Prague. The accidents and closures are time-limited restrictions and so their detection is a difficult task. Strategies of the traffic control have been developed for more than 40 years. The first designed traffic controls were fixed control strategies (Papageorgiou et al., 2003). Fixed control strategies are based on a signal-timing plan defined by a typical daily traffic courses. Such control does not require any traffic detectors at the intersection. This type of control can be found mainly in smaller towns. 
However, the pre-determined settings are not sufficient. It was found that the capacity of traffic network increases markedly when the traffic control via the signal lights is changing according to the current traffic situation. The newer approaches, so-called dynamic traffic control strategies, model the traffic situation from real time or near real time traffic information .

The first dynamic control strategies were designed to control of a single intersection only. Such controller does not co-operate with controller of the adjacent intersections. Uncoordinated traffic control can cause traffic congestion. New solution of the traffic control co-operates between adjacent intersections controllers. It means that e. g. the green wave is keeped. Around the world, several types of dynamic traffic control strategies are used. The most often used strategies are: TRANSYT (Courage, K. and Wallace, C.E., 1991; Robertson, 1969; ,Wong et al., 2000), SCOOT, SCATS (Hunt et al., 1982; Lowrie, P. R., 1982; Moor et al., 2005; Kosmatopoulos et al., 2006; Chilukuri et al., 2004) and MOTION (Pearson, 2000; Tichý, 2007). The strategy TRANSYT is based on principle of minimisation of a special criterion characterising the traffic flow. Then, the optimal signal plan is chosen from set of predefined signal plans according to the criterion value. The strategies SCOOT and SCATS are available for high-capacity traffic networks where each road has several lanes. The strategy MOTION is based on adaptive principle and it is designed for full co-operation between intersections. Also several other traffic control strategies have been developed, e.g. PRODYN, RHODES, TUC (Papageorgiou et al., 2003).

All types of dynamic traffic control strategies need information about the current traffic situation, i.e. about the actual traffic flow. The current traffic situation can be monitored by using several types of measurement devices, e.g. inductive detectors ${ }^{1}$, radars and video detectors. Inductive detectors are placed under the road surface and they are activated/deactivated by passing vehicles. Radars and video detectors are usually used for detection and monitoring of vehicles at intersections. These types of traffic detectors require a long visual range and their acquisition costs are high. This limitations lead to the usage of the inductive detectors in the cities and aerial detectors are mostly used on motorways.

Most of the previously introduced dynamic control strategies are commercial tools with all associated advantages and disadvantages. The main advantage is in availability of full service. The main disadvantage is problematic adaptation of such product to the needs of a particular customer. These commercial strategies are usually geared to traffic control of big cities where the traffic network consists of the multilanes and the capacity of traffic network is huge. In the historical cities, such as Prague, these control strategies cannot be employed without problems (for example, application of the traffic control system MOTION in Prague shows its sensitivity on driver habits (Tichý, 2007)). For this reason, in Prague, a new control

\footnotetext{
1 The road (or lane) can be equipped with one detector on the output and three detectors on the input: (i) detector on the stop line, (ii) outlying detector ( $30 \mathrm{~m}$ before the stop line), (iii) strategic detector - (100 m before the stop line). Ideally, each lane has all three types of the detectors but in real traffic system, the lane is usually equipped with one or two types of such detector, due to the constrained conditions e.g. in the old urban areas with short lanes. The strategic detectors, which are the most remote from a stop line, give the best information about the traffic flow at present. Unfortunately, the detector cannot provide any useful information about the real traffic flow if the queue length is greater then the distance between stop line and the detector placement.
} 
strategy is being designed (Kratochvílová, 2004; Homolová, 2005; Pecherková et al., 2006). This traffic control strategy has been designed especially for historical urban areas, characteristic by a traffic network formed from many narrow one-way roads which are equipped mainly by the inductive detectors.

The designed traffic control strategy is based on the principle of sum of queue lengths minimization. The queue lengths are hardly measurable and predictable quantities. Currently, this quantity can be consider as unmeasured. For this reason, the queue lengths have to be estimated from available data by a suitable estimation method on the basis of proper traffic model. The main problem is thus to specify the exact model of the traffic flow behaviour on intersection or micro-region. The traffic model is considered as a non-linear state space model, where the quantity representing the queue lengths belongs to the state. Due to the nonlinearities in the state equation, the traffic system state is estimated by means of the nonlinear estimation methods on the basis of quantities such as intensity of the traffic flow or the occupancy measured by the detectors.

The main aim of this article is twofold. First, the focus will be directed to the design and indepth description of the traffic system model. This model should be designed to properly describe the behaviour of a traffic flow on an arbitrarily complex micro-region. Second, the designed model will be validated and thus the methods for the model validation will be presented and applied.

The chapter is organised as follows. Section 2 provides a complete description of traffic model and its design. Section 3 deals with verification of a traffic model and there will be a short overview of suitable estimation methods employed for validation. In Section 4, the experiments presenting the properties and validation of the designed model using the real and the synthetic data will be shown. Finally, Section 5 comprises concluding remarks.

\section{Traffic model design}

This section will be devoted to the description of the traffic system model. First, basic quantities that describe the traffic system will be introduced. Second, the model of the traffic system based on the conservation principle will be presented. The description of the model will start with the case of a simple microregion and then it will be generalized.

\subsection{Traffic quantities}

There are many quantities that characterize the traffic system. These quantities can be divided into two basic groups:

i. Quantities determined by the intersection layout and configuration

- Saturation flow $\mathcal{S}_{k}$ corresponds to the maximal number of vehicles flowing through the intersection arms per hour - given in $[u v / h]$, where $u v$ represents a unit vehicle. This quantity mainly depends on the road width, number of traffic lanes in one direction, and turning movement.

- Turning movement $\alpha_{i, j}$ is the ratio of vehicles going from the $i^{\text {th }}$ arm to the $j^{\text {th }}$ arm [\%].

- Cycle time $t_{c}$ is a period of a phase change of the traffic light [s].

- Green time ratio $z_{k}$ is the ratio of the effective green time to the measurement time period. Note that the green time ratio is usually defined as a ratio of the effective green time to the cycle time (Ackerman, 2000). However, such definition can lead to the possible discrepancy between the cycle time and measurement period which can lead to significant problems. 
- Offset is the difference between the start (or end) of green at the two adjacent signalized intersection $[s]$.

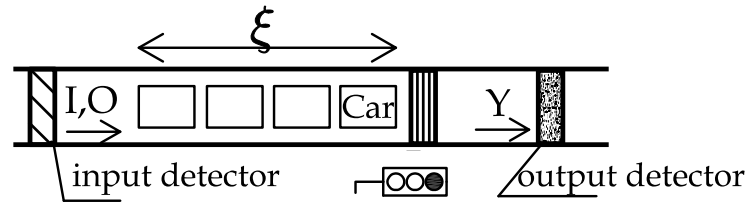

Fig. 1. Example of one way-road.

ii. Quantities describing the traffic flow

- Input intensity $\mathcal{I}_{k}$ or output intensity $\mathcal{Y}_{k}$ (at time instant $k$ ) is the amount of passing unit vehicles per hour $[u v / h]$ measured by the detector placed in the input or output lane, respectively.

- Occupancy $O_{k}$ is the proportion of the period when the detector is occupied by vehicles [\%].

- Queue length $\xi_{k}$ is a maximal number of vehicles waiting in one lane at period $[u v / \text { period }]^{2}$.

Although, majority of quantities in the previous list is assumed as units per hour, in the designed traffic model all these quantities are recalculated with respect to the respective time period of the measurement. It means, that saturation flow $S_{k}$ is given in unit vehicle per hour $([u v / h])$, but in the model the corresponding recalculated quantity $S_{k}$ is given in unit vehicle per period ([uv/period] $]$. The same goes for the input intensity $\mathcal{I}_{k}$ and the output intensity $y_{k}$, respectively. As the model is discrete then the dependence on period can be omitted, i.e. the quantities are given only in their respective units.

\subsection{Traffic model}

The fundamental idea of the described traffic model design technique is based on the traffic flow conservation principle. It means that the queue at time $k+1$ is equal to the sum of the previous queue at time $k$ and input intensity minus output intensity from the arm.

\section{Simple micro-region}

For the sake of simplicity the proposed technique for traffic system modelling will firstly be shown on the simple micro-region. This micro-region comprises a road with one input and one output detector and one traffic light as it is depicted in Figure 1. The traffic situation at time instant $k$ is completely described with the state $x_{k}$. The state $x_{k}$ is formed from the queue length $\xi_{k}$, the input intensity $I_{k}$ and the occupancy $O_{k}$. The measurement vector $y_{k}$ is in the considered micro-region formed from the input intensity $I_{k}$ and occupancy $O_{k}$ measured on the strategic detector, the output intensity $Y_{k}$ measured on output detector and the intensity $I_{k}^{S L}$ measured on the stop-line detector.

The state-space model is given as follows:

2 The queue length can be also considered in meters. The length of unit vehicle is supposed to be $6 \mathrm{~m}$. 


$$
\begin{gathered}
x_{k+1}=\left[\begin{array}{l}
x_{1, k+1} \\
x_{2, k+1} \\
x_{3, k+1}
\end{array}\right]=\left[\begin{array}{c}
\xi_{k+1} \\
I_{k+1} \\
O_{k+1}
\end{array}\right]=\left[\begin{array}{c}
\xi_{k}+I_{k}-I_{k}^{\pi}\left(\xi_{k}, I_{k}, V_{k}\right)+w_{1, k} \\
I_{k}+w_{2, k} \\
\kappa_{k} \xi_{k}+\beta_{k} O_{k}+\lambda_{k}+w_{3, k}
\end{array}\right]= \\
=\left[\begin{array}{c}
x_{1, k}+x_{2, k}-I_{k}^{\pi}\left(x_{1, k}, x_{2, k}, V_{k}\right) \\
\left.x_{2, k}\right) \\
\kappa_{k} x_{1, k}+\beta_{k} x_{3, k}+\lambda_{k}
\end{array}\right]+\left[\begin{array}{l}
w_{1, k} \\
w_{2, k} \\
w_{3, k}
\end{array}\right], \\
y_{k}=\left[\begin{array}{l}
x_{2, k} \\
y_{2, k} \\
y_{3, k} \\
y_{4, k}
\end{array}\right]=\left[\begin{array}{c}
I_{k} \\
O_{k} \\
Y_{k} \\
I_{k}^{S L}
\end{array}\right]=\left[\begin{array}{c}
x_{3, k} \\
I_{k}^{\pi}\left(x_{1, k}, x_{2, k}, V_{k}\right) \\
I_{k}^{\pi}\left(x_{1, k}, x_{2, k}, V_{k}\right)
\end{array}\right]+\left[\begin{array}{l}
v_{1, k} \\
v_{2, k} \\
v_{3, k} \\
v_{4, k}
\end{array}\right] .
\end{gathered}
$$

The queue length $\xi_{k+1}$ is given by the queue at previous time instant $\xi_{k}$, the input intensity $I_{k}$ representing the number of arrived cars and by the function $I_{k}^{\pi}\left(\xi_{k}, I_{k}, V_{k}\right)$ describing the number of passing vehicles. The occupancy $O_{k+1}$ depends on the occupancy and the queue length at previous time instant and on the parameters $\kappa_{k}, \beta_{k}$, and $\lambda_{k}$ which cannot be exactly determined from physical properties of a micro-region and they are generally unknown. The remaining state variable $I_{k}$ is modelled as a random walk. The probability density functions (pdf's) of the state noises $w_{i, k}$ and measurement noises $v_{i, k}$ are currently supposed to be zero mean with unknown covariance matrices. The state and measurement noises are supposed to be mutually independent and independent of the traffic system initial state. Note that in this simple example the measured intensity on the output detector $Y_{k}$ and on the stop-line detector $I_{k}^{S L}$ are the same. These intensities are different for microregions with more than one arm.

The function $I_{k}^{\pi}\left(\xi_{k}, I_{k}, V_{k}\right)$ represents the number of departed vehicles and depends on three quantities: (i) the queue length $\xi_{k}$, (ii) the input intensity $I_{k}$ and (iii) the maximal number of passing vehicles $V_{k}$ which can pass through the intersection in the measurement period. In short, the function represents a continuous approximation of the theoretical throughput of the intersection as it is shown in Figure 2. For details, see (Pecherková et al., 2007). The function $I_{k}^{\pi}\left(\xi_{k}, I_{k}, V_{k}\right)$ has following form

$$
I_{k}^{\pi}\left(\xi_{k}, I_{k}, V_{k}\right)=V_{k} \cdot\left(1-e^{-\frac{1}{V_{k}}\left(\xi_{k}+I_{k}\right)}\right)=V_{k} \cdot\left(1-e^{-\frac{1}{V_{k}}\left(x_{1, k}+x_{2, k}\right)}\right)
$$

The maximal number of passing vehicles $V_{k}$ is given by the saturation flow $S_{k}$ and the green time ratio $z_{k}$. The quantity $V_{k}$ can be written as

$$
V_{k}=S_{k} \cdot z_{k}
$$




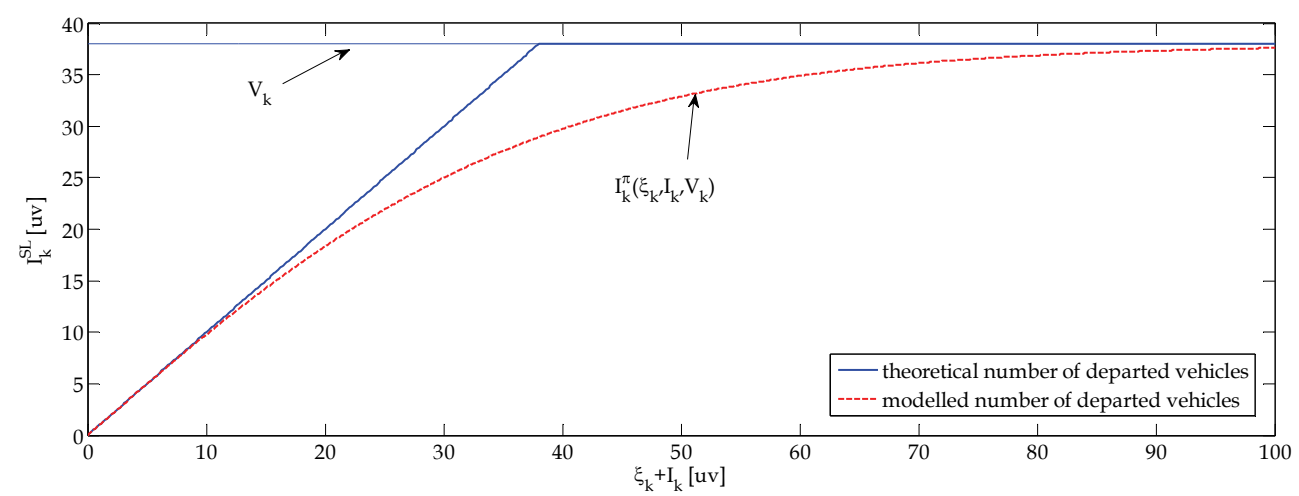

Fig. 2. Theoretical and modelled number of the departed vehicles.

In equation (4), it can be seen that saturation flow $S_{k}$ is supposed to be time-variant. In majority of the traffic control strategies, the saturation flow is assumed as time-invariant because working with time-variant one is more complicated. In this chapter, the saturation flow is assumed to be time-variant. Precondition of the time-invariant saturation flow is inaccurate because the actual saturation flow depends on the both invariant and timevariant quantities. The following relation shows how the saturation flow at time instant $k$ will be evaluated

$$
S_{k}=\left(S_{0}-\alpha^{R} c_{1, k}-\alpha^{L}\left(c_{2, k}+c_{3, k} I_{k}^{P}\right)\right) \cdot \gamma_{k} \cdot P H F .
$$

The actual saturation flow $S_{k}$ depends on the theoretic saturation flow $S_{0}$, ratio of heavy vehicles $\gamma_{k}$ in the average traffic flow, right and left turning movements $\alpha^{R}$ and $\alpha^{L}$, respectively, the parameters of the traffic flow behaviour $c_{1, k}, c_{2, k}, c_{3, k}$, intensity of oncoming vehicles $I_{k}^{P}$ and on peak hour factor PHF.

The theoretic saturation flow $S_{0}$ is time-invariant and depends on width of roads, speed limit and the shape of an intersection. The time-variant turning movements are not directly measurable. However, it is possible to find their typical daily values by means of analysis of measured and simulated data.

The last time-invariant traffic quantity is the peak hour factor PHF. This factor respects the fact that in oversaturation the vehicles have to start, stop and brake more often than usually. This behaviour reduces the speed of the traffic flow and the capacity of the road.

The ratio of heavy vehicles $\gamma_{k}$ and parameters of the traffic flow behaviour $c_{1, k}, c_{2, k}, c_{3, k}$ belong to the group of time-variant quantities and parameters. The parameter $\gamma_{k}$ describes the ratio between heavy and light vehicles (Kara, K. \& Shabin, R., 2000). Heavy vehicles have different driving properties than light vehicles and so the traffic flow has different behaviour for the different ratios. The ratio of heavy vehicles $\gamma_{k}$ is an unmeasured quantity but unlike turning movement, the changes are not so significant and so it is possible to estimate this factor relatively well. The parameters of the traffic flow behaviour $c_{1, k}, c_{2, k}$ 
and $c_{3, k}$ can compensate faulty estimation of the actual turning movements. This fault is caused by precondition of time-invariant turning movement. These three parameters model the reduction of speed and the number of passing vehicles according to the turning of vehicles. The parameter $c_{1, k}$ models the right turning movement and the apriori setting of this parameter is given by typical turning movement and radius of right turn. The same function has parameter $c_{2, k}$ which models the left turning movement. The last parameter $c_{3, k}$ models intensity of oncoming vehicles with respect to the left turning movement. For example, strong left turning movement in combination with high oncoming intensity can cause saturation flow to be several times smaller than the theoretic saturation flow. In the micro-region described by relations (1) and (2), the actual saturation flow is identical to theoretical saturation flow since no turning movements and oncoming intensities.

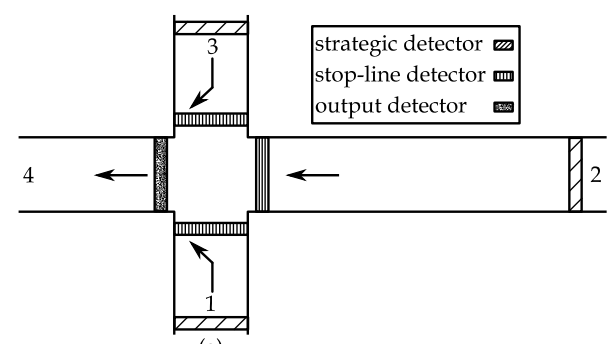

(a)

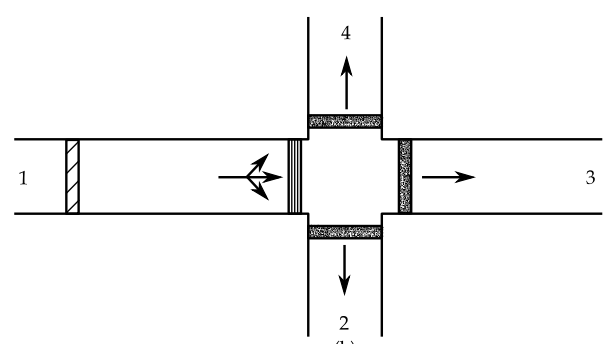

(b)

Fig. 3. Outline of more complex micro-regions: (a) four arms intersection with three input arms and one output arm, (b) four arms intersection with one input arm and three output arms.

\section{More complex micro-regions}

To illustrate the situation when either the actual and theoretical saturation flows are not the same or the intensities $I_{k}^{S L}$ and $Y_{k}$ are different, the model design for two other microregions will be shown. The first micro-region consists of one intersection with three input arms and one output arm, see Figure 3a. The second micro-region consists of one intersection with one input arm and three output arms, see Figure $3 \mathrm{~b}$. All arms are one-way with one lane only.

The first micro-region consists of one intersection with three input arms and one output arm. The arms number 1, 2 and 3 are the inputs arms and the arm number 4 is the output arm. This system is not uncommon in historical centres of citites. Such system can work with two- or three-phrase control. In this case, the two-phase control is used, in the first phase the arms number 1 and 3 have green simultaneously and in the second phase the arm 2 has green. The influence of the green time on the traffic model is not seen in the state or the measurement relations explicitly. The green time influences the nonlinear function $I_{k}^{\pi}\left(\xi_{k}, I_{k}, V_{k}\right)$ and is used for computation of the quantity $V_{k}$.

The construction of the model of such micro-region is based on the modelling of each arm separately and then on the modelling of particular relations among all arms. In this particular micro-region, the model has the dimension of the state $\operatorname{dim}\left(x_{k}\right)=9$ and 
dimension of measurements $\operatorname{dim}\left(y_{k}\right)=10$. The relation describing the state update is then given as

$$
x_{k+1}=\left[\begin{array}{c}
x_{1, k+1} \\
x_{2, k+1} \\
x_{3, k+1} \\
x_{4, k+1} \\
x_{5, k+1} \\
x_{6, k+1} \\
x_{7, k+1} \\
x_{8, k+1} \\
x_{9, k+1}
\end{array}\right]=\left[\begin{array}{c}
\xi_{1, k+1} \\
\xi_{2, k+1} \\
\xi_{3, k+1} \\
I_{1, k+1} \\
I_{2, k+1} \\
I_{3, k+1} \\
O_{1, k+1} \\
O_{2, k+1} \\
O_{3, k+1}
\end{array}\right]=\left[\begin{array}{c}
x_{1, k}+x_{4, k}-I_{1, k}^{\pi}(\cdot)+w_{1, k} \\
x_{2, k}+x_{5, k}-I_{2, k}^{\pi}(\cdot)+w_{2, k} \\
x_{3, k}+x_{6, k}-I_{3, k}^{\pi}(\cdot)+w_{3, k} \\
x_{4, k}+w_{4, k} \\
x_{5, k}+w_{5, k} \\
x_{6, k}+w_{6, k} \\
\kappa_{1, k} x_{1, k}+\beta_{1, k} x_{7, k}+\lambda_{1, k}+w_{7, k} \\
\kappa_{2, k} x_{2, k}+\beta_{2, k} x_{8, k}+\lambda_{2, k}+w_{8, k} \\
\kappa_{3, k} x_{3, k}+\beta_{3, k} x_{9, k}+\lambda_{3, k}+w_{9, k}
\end{array}\right]
$$

and the measurement vector is given as

$$
y_{k}=\left[\begin{array}{c}
y_{1, k} \\
y_{2, k} \\
y_{3, k} \\
y_{4, k} \\
y_{5, k} \\
y_{6, k} \\
y_{7, k} \\
y_{8, k} \\
y_{9, k} \\
y_{10, k}
\end{array}\right]=\left[\begin{array}{c}
I_{1, k} \\
I_{2, k} \\
I_{3, k} \\
O_{1, k} \\
O_{2, k} \\
O_{3, k} \\
Y_{4, k} \\
I_{1, k}^{S L} \\
I_{2, k}^{S L} \\
I_{3, k}^{S L}
\end{array}\right]=\left[\begin{array}{c}
x_{4, k}+v_{1, k} \\
x_{5, k}+v_{2, k} \\
x_{6, k}+v_{3, k} \\
x_{1, k}+v_{4, k} \\
x_{7, k}+v_{5, k} \\
x_{8, k}+v_{6, k} \\
\alpha_{1,4} I_{1, k}^{\pi}(\cdot)+\alpha_{2,4} I_{2, k}^{\pi}(\cdot)+\alpha_{3,4} I_{3, k}^{\pi}(\cdot)+v_{7, k} \\
I_{1, k}^{\pi}(\cdot)+v_{8, k} \\
I_{2, k}^{\pi}(\cdot)+v_{9, k} \\
I_{3, k}^{\pi}(\cdot)+v_{10, k}
\end{array}\right] .
$$

The intensities $I_{i, k}^{\pi}(\cdot)$ represent the number of passing vehicles from the $i^{\text {th }}$ arm where $i=1,2,3$ and the turning movements $\alpha_{i, 4}$ are equal to one because the traffic flow does not divide into several streams.

The second micro-region describes more usual situation where the traffic flow from one arm is divided into several streams. In this traffic system, the input intensity $I_{k}$ is divided into three output intensities $Y_{1, k}, Y_{2, k}$ and $Y_{3, k}$ according to particular turning movements $\alpha_{i, j}$. The subscript $i$ denotes the number of the input arm (in this case, $i=1$ ) and $j$ the number of output arm.

In this case, the state update and measurament are described by the relations

$$
x_{k+1}=\left[\begin{array}{l}
x_{1, k+1} \\
x_{2, k+1} \\
x_{3, k+1}
\end{array}\right]=\left[\begin{array}{c}
\xi_{1, k+1} \\
I_{1, k+1} \\
O_{1, k+1}
\end{array}\right]=\left[\begin{array}{c}
x_{1, k}+x_{2, k}-I_{1, k}^{\pi}(\cdot)+w_{1, k} \\
x_{2, k}+w_{2, k} \\
\kappa_{1, k} x_{1, k}+\beta_{1, k} x_{3, k}+\lambda_{1, k}+w_{3, k}
\end{array}\right]
$$




$$
y_{k}=\left[\begin{array}{c}
y_{1, k} \\
y_{2, k} \\
y_{3, k} \\
y_{4, k} \\
y_{5, k} \\
y_{6, k}
\end{array}\right]=\left[\begin{array}{c}
I_{1, k} \\
O_{1, k} \\
Y_{2, k} \\
Y_{3, k} \\
Y_{4, k} \\
I_{1, k}^{S L}
\end{array}\right]=\left[\begin{array}{c}
x_{2, k}+v_{1, k} \\
x_{3, k}+v_{2, k} \\
\alpha_{1,2} I_{1, k}^{\pi}(\cdot)+v_{3, k} \\
\alpha_{1,3} I_{1, k}^{\pi}(\cdot)+v_{4, k} \\
\alpha_{1,4} I_{1, k}^{\pi}(\cdot)+v_{5, k} \\
I_{1, k}^{\pi}(\cdot)+v_{6, k}
\end{array}\right]
$$

The turning movements $\alpha_{i, j}$ influence not only the output intensity but also the saturation flow. The turning movements are assumed to follow the typical daily values and their sum have to be equal to one.

The previously described micro-regions can form together typical two-ways four-arm intersection with one lane in each direction. In such a case, the resulting model is obtained by simple coupling of the two previous models, i.e the three input arms with one output arm and one input arm with three output arms. Such traffic model has then the dimension of state $\operatorname{dim}\left(x_{k}\right)=12$ and dimension of the measurement $\operatorname{dim}\left(y_{k}\right)=16$. Usually the microregion consists of 3 - 4 four-arm controlled intersections and equal number of uncontrolled intersections. Typically, uncontrolled intersections are not equipped with the detectors and so the intensities and occupancies are unmeasured quantities and they have to be estimated as well as queue lengths. Note that more complex micro-region will be discussed in Section 4 .

\section{Validation of the traffic model}

In the previous section, a technique for the model design of an arbitrary micro-region was introduced. The aim of this section is to present procedures suitable for validation of the traffic system models.

Two criteria for validation of the models designed by means of the proposed technique are considered. The first one compares the "true" system state with its estimate. The second criterion compares the measured and predicted system output.

\subsection{Validation via state estimation}

The validation is based on the comparison of the true state of the traffic system with its estimate. The true state of the traffic system, particularly the unknown part of the true state representing the immeasurable queue lengths, can be determined by simulation software AIMSUN $^{3}$. The estimates of the traffic system state can be found using various estimation techniques. However, the quality of the state estimates produced by the techniques strongly

${ }^{3}$ AIMSUN is a simulation software tool which is able to reproduce the traffic condition of any traffic network. It is mainly used for testing new traffic control system and management strategies, but it can be also used for traffic state prediction and other real time applications. The validation and calibration process of the simulator was made with respect to the particularities of the local traffic system. The validation of the queue length reconstruction was made on several types of micro-regions in Prague in accordance to the guidelines specified in AIMSUN (AIMSUN: Users manual, 2004). 
depends on the accuracy of the traffic system model. The considered state estimation techniques are briefly described in the following text.

The aim of the state estimation is to find an estimate of the state $x_{k}$ conditioned by the measurements $y(k)=\left[y_{0}, y_{1}, \ldots, y_{k}\right]$ up to the time instant $k$. The state estimate is usually given by the conditional pdf $p\left(x_{k} \mid y(k)\right)$ or by the conditional mean $\hat{x}_{k \mid k}=E\left[x_{k} \mid y(k)\right]$ and covariance matrix $P_{k \mid k}=\operatorname{cov}\left[x_{k} \mid y(k)\right]$.

Utilisation of the state estimation methods is conditioned by the complete knowledge of the model. However, the nonlinear model presented in previous section contains several unknown parameters, in both the "deterministic" and the "stochastic" part, which cannot be determined from the physical properties of the traffic system, namely parameters $\beta_{k}, \kappa_{k}, \lambda_{k}$ and the statistics of the state and measurement noises. Therefore, the unknown parameters have to be identified somehow.

Generally, there are two possibilities how to estimate the state and the parameters in the deterministic part of the system. The first possibility is based on an off-line identification of the unknown parameters, e.g. by prediction error methods (Ljung, 1999), and subsequently on an on-line estimation of the state by the nonlinear state estimation techniques. However, off-line identified parameters represent average values rather than the actual (true) parameters and this approach is therefore suitable for traffic systems where intensity of the traffic flow is almost constant. The second possibility is based on the simultaneous on-line estimation of the state and the parameters by extension of the state with vector of the unknown parameters (Wan et al., 2000). This leads to the extended nonlinear model of the traffic.

There are two main groups of the nonlinear estimation methods, namely local and global methods. Although, the global methods are more sophisticated than local methods, they have significantly higher computation demands. Due to the computational efficiency, the stress will be mainly laid on the derivative-free local filter method, namely the divided difference filter first order (Nørgaard et al., 2000). The comparison of various other local filtering methods (Julier \& Uhlmann, 2004; Mihaylova et al., 2006; Hegyi at al., 2006) in traffic area can be found in e.g. (Pecherková et al., 2007).

The application of the local methods is also conditioned by the knowledge of the secondorder statistics of the state noise $w_{k}$ and the measurement noise $v_{k}$. The state and measurement noise covariance matrices, can be hardly determined from the physical properties of the traffic system and they have to be estimated somehow. The noise covariance matrices can be generally estimated online or offline. Due to the extensive computational demands of online noise covariance matrices estimation methods (Mehra, 1972; Verdú \& Poor, 1984), they were estimated offline by the estimation technique based on the multi-step prediction (Šimand \& Duník, 2008) for both nonlinear models and extended nonlinear models for various intensities of the traffic flow.

Note that the state estimation techniques with state inequality constrains were used (Simon \& Simon, 2003; Simon \& Chia, 2002) in order to ensure that the state quantities are in an admissible region of the state space. The admissible region is defined on the basis of the physical consideration, for example the queue lengths cannot be negative.

The true and estimated queue lengths are compared via the root mean square error criterion 


$$
J^{S}=\sqrt{\frac{\sum_{k=0}^{K} \sum_{i=1}^{n_{q}}\left(x_{i, k}-\hat{x}_{i, k \mid k}\right)}{(K+1) \times n_{q}}}
$$

where $n_{q}$ is the number of estimated queue lengths, $x_{i, k}$ represents the $i^{\text {th }}$ component of the state, $\hat{x}_{i, k \mid k}$ its filtering estimate, and $K$ is the number of measured data. Thus, the $J^{S}$ represents an average error of the queue length estimate on one arm at one sample period. The value of the criterion depends not only on the applied estimation technique, but also on the quality of the proposed traffic model.

\subsection{Validation via system output prediction}

The state estimate $\hat{x}_{k-t \mid k-t}$ can be used to compute the $t$-step prediction of the output $\hat{y}_{k \mid k-t}$. The multi-step prediction can be obtained as multiple application of the one-step prediction which is an essential part of the state estimation algorithms (Šimand \& Duník, 2006).

The multi-step prediction of the output depends not only on the measured output data up to the time instant $k-t$, i.e. $y(k-t)=\left[y_{0}, y_{1}, \ldots, y_{k-t}\right]$, and measured input data up to the time instant $k$, i.e. $u(k)$, but it also strongly depends on the quality of the model. Thus, the quality of the traffic system output can be validated also according to the following criterion

$$
J_{y_{i}, t}^{P}=\frac{\sum_{k=t}^{K}\left(y_{i, k}-\hat{y}_{i, k \mid k-t}\right)}{(K-t+1) \times n_{y}},
$$

where $y_{i, k}$ represents the $i^{\text {th }}$ component of the measurement and $\hat{y}_{i, k \mid k-t}$ is the $t$-step prediction of $y_{i, k}$. The criterion compares the measured data with predicted data, which are computed according to the model.

\section{Experiments}

In previous two sections the traffic model design technique was introduced as well as the procedures for the model validation. The aim of this section is to demonstrate the behaviour and validity of the two designed models.

The real data, namely the input and output intensities, the occupancies and the cycle and green times, were collected on real places in the Prague traffic system. However, for the model validation the queue lengths are also needed. The queue lengths cannot be directly measured and thus they were syntetized using calibrated traffic simulator AIMSUN.

First, the validation of model of one way four-arm intersection where one arm is input arm and another arms are output arms will be presented. Second, typical two-way four-arm 
intersection will be shown. This type of intersection is very frequent intersection in the traffic networks all over the world.

\subsection{One-way four-arm intersection}

One-way four-arm intersection can be designed in different combinations of inputs and outputs. In the particlar case of this example, it is assumed that this intersection has one input and three ouput detectors, see Figure $3 \mathrm{~b}$. The basic model describing this intersection is given by the equations (8) and (9). For estimation and prediction the model is augmented with the unknown time-variant traffic parameters $\kappa_{1, k}, \beta_{1, k}$ and $\lambda_{1, k}$ and uknown traffic flow behaviour parameters $c_{1, k}, c_{2, k}$ and $c_{3, k}$.

This example demonstrates the quality of the model in case of the standard traffic flow on the arm 1, i.e without congestion. The model of this intersection will be validated using the criteria (10) and (11). The criterion $J^{S}$ evaluates only the root mean square error between the true and the estimated queue lengths on arm 1 . The criterion $J^{P}$ is evaluated for the following quantities:

- The input intensity $I_{1}$ and its one-step prediction.

- The input occupancy $O_{1}$ and its one- and two-step prediction.

- The intensity on the stop-line $I_{1}^{S L}$ and its one-step prediction.

- The output intensity $Y_{2}$ and its one-step prediction.

The resulting criteria values are presented in Table 1 . This table shows the actual values of the criterion accompanied with the maximal values. The maximal values are given in order to illustrate the scale of the errors with respect to the actual amplitudes of the corresponding quantities.

\begin{tabular}{|c|c|c|c|c|c|c|}
\hline & $J_{I_{1}=y_{1}, t=1}^{P}$ & $J_{O_{1}=y_{2}, t=1}^{P}$ & $J_{O_{1}=y_{2}, t=2}^{P}$ & $J_{I_{1}^{S L}=y_{3}, t=1}^{P}$ & $J_{Y_{2}=y_{4}, t=1}^{P}$ & $J^{S}$ \\
\hline$J$ & 0.4331 & 0.0805 & 0.1027 & 0.9783 & 1.3279 & 3.5897 \\
\hline Max. value of $y_{i}$ or $x_{i}$ & 19 & 18.08 & 18.08 & 21 & 6 & 40 \\
\hline
\end{tabular}

Table 1. The values of criterion (10) for the input intensity $I_{1}$, output occupancy $O_{1}$, output intensity $Y_{2}$ and the intensity on the stop-line $I_{1}^{S L}$ and criterion (11) for the queue length. The last row contains maximal values of the corresponding quantities in the state or measurement.

Figure 4 depicts the typical daily courses of the queue length and occupancy on the arm 1 . From the figure and from the ratio of the criterion and maximal value in Table 1 it can be seen that the quality of the estimates based on the designed model is adequate. So the model (8), (9) sufficiently accurately describes the considered micro-region.

\subsection{Two four-arm intersections}

In the second example the micro-region comprises two interconnected four-arm intersection. Each of the intersection arms is two-way road. The micro-region is outlined in Figure 5. The model of each intersection in this micro-region is given by simple coupling of the two models described by relations (6), (7) and (8), (9). The output quantities on the arm 3 are 
equal to the input quantities on the arm 5 and vice versa. Again as in the previous example the model is augmented with the unknown time-variant traffic parameters.
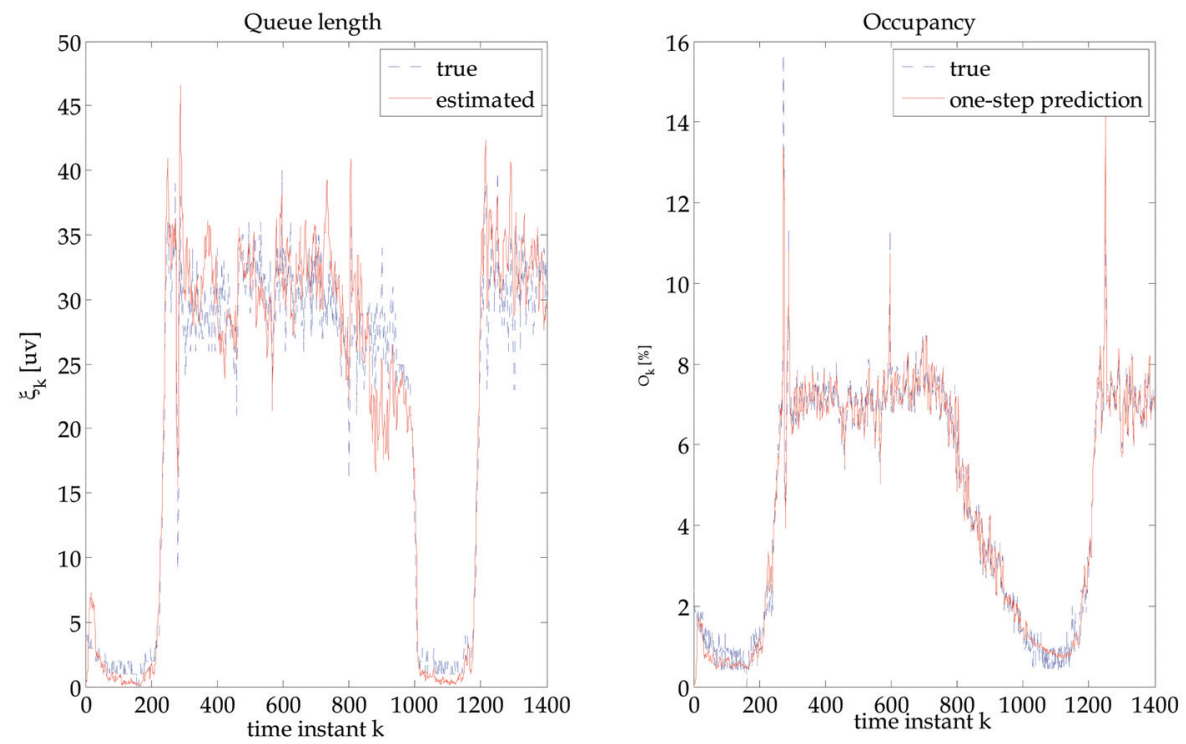

Fig. 4. An example of the true and estimated queue lengths and the true and predicted occupancy for the intersection arm 1.

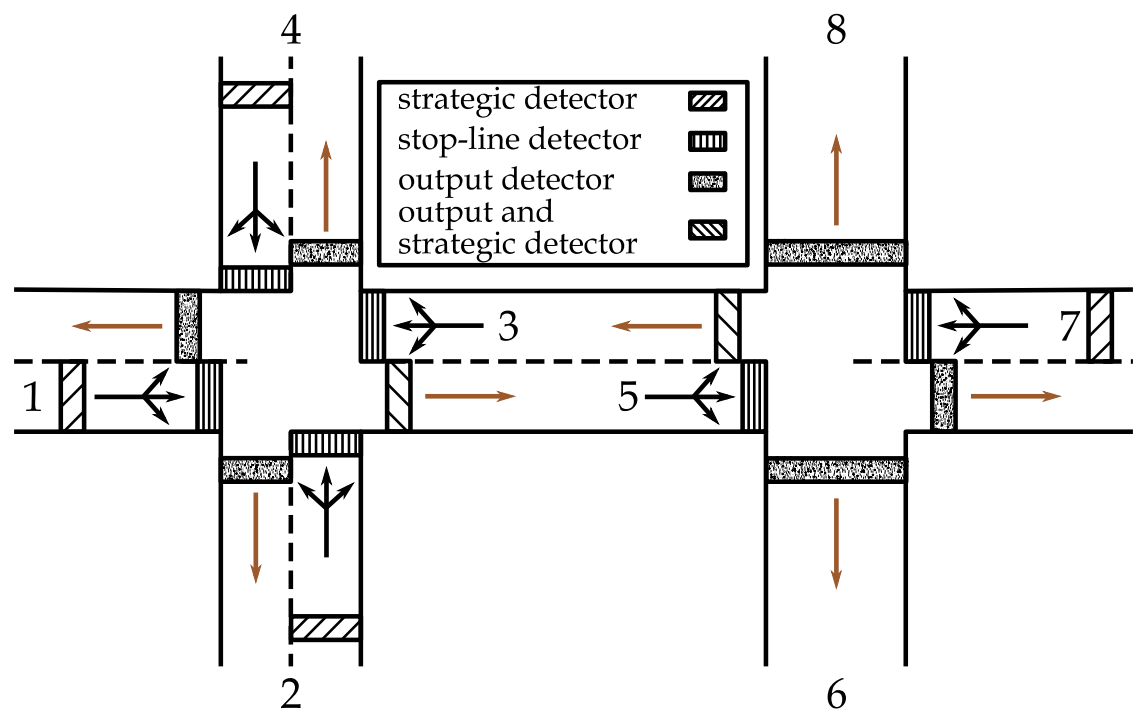

Fig. 5. Outline of two four-arm intersection. 

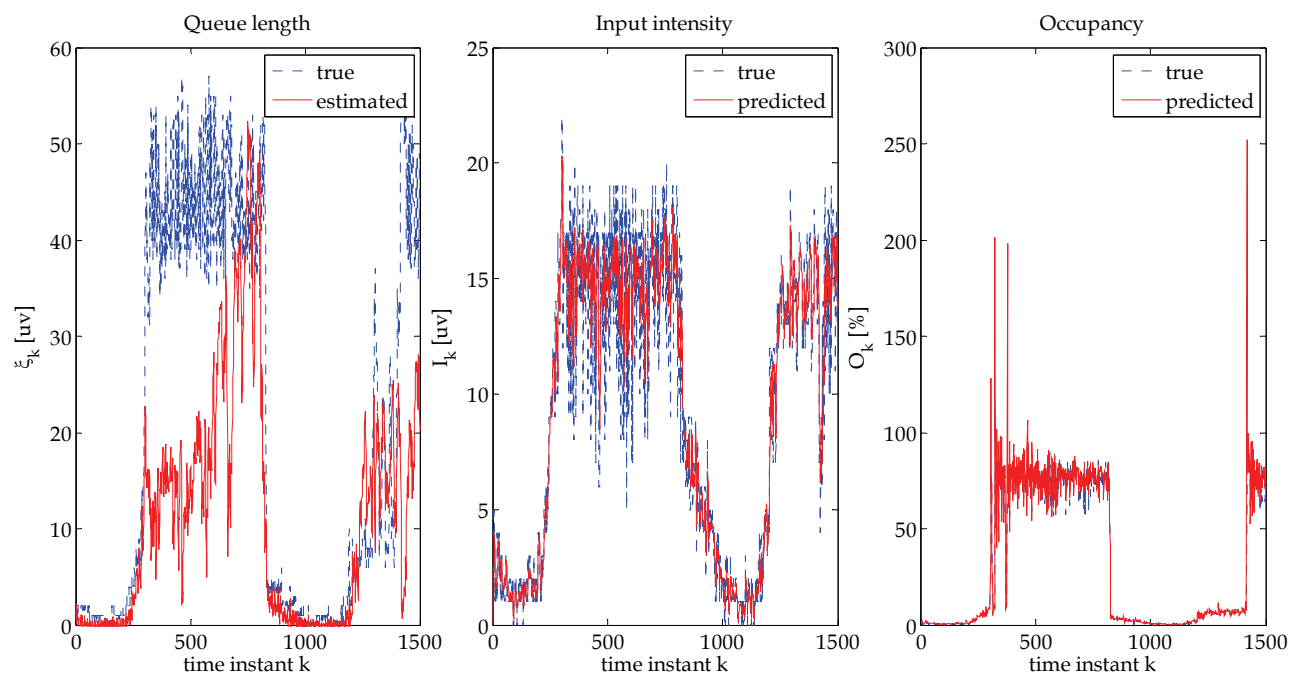

Fig. 6. The true and estimated queue length and the true and predicted input intensity and occupancy, respectively, on arm 1 (in the oversaturated situation the queue reaches over the strategic detector).
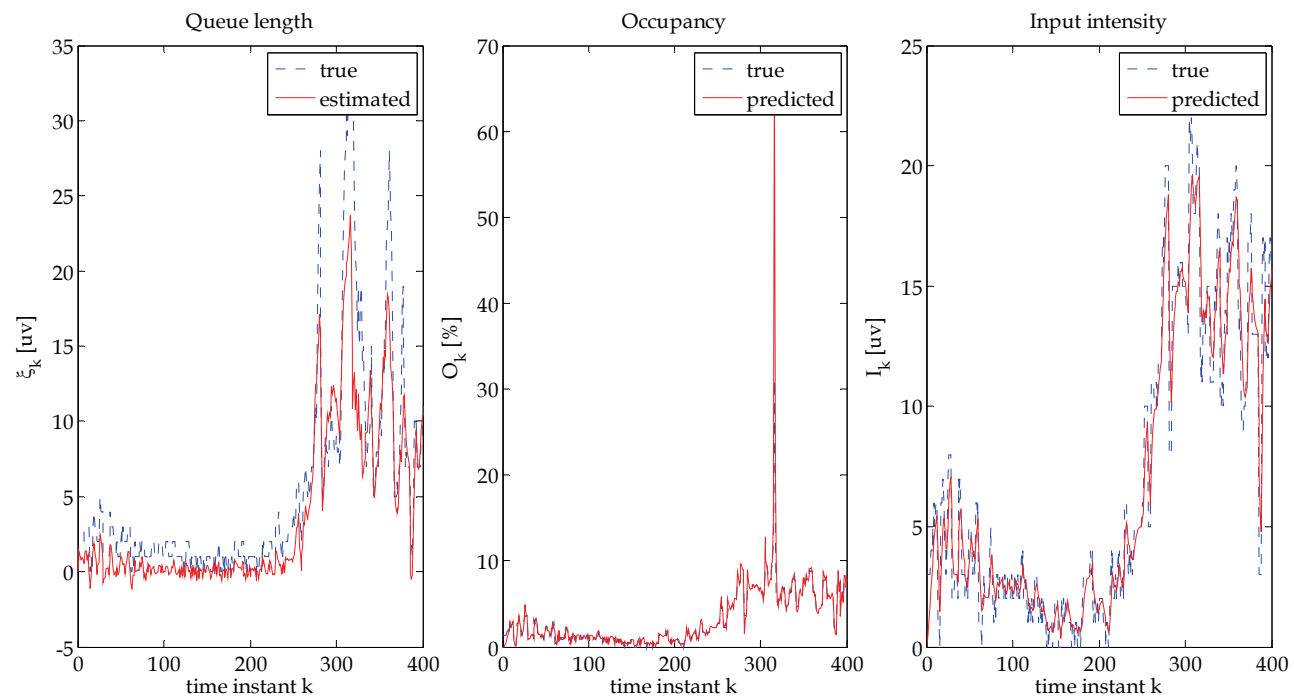

Fig. 7. The true and estimated queue length and the true and predicted input intensity and occupancy, respectively, on arm 3 (standard traffic situation, i.e. without oversaturation).

In this case some of the quantities do not always have the standard behaviour. On arms 1 and 7 the oversaturated traffic flow is occurring, i.e. the real queue often reaches over the strategic detector. This exceptional state can be verified on the basis of the occupancy. If the 
occupancy is higher than $70-80 \%$ then the traffic flow can be considered to be oversaturated (Gazis, D. C., 1964; Green, D. H., 1967). Then, the quantities measured by the traffic detectors do not contain any information about the real number of arrived vehicles. Therefore, the quality of the estimated and predicted quantities is significantly worsened. The situation with oversaturated traffic flow on arm 1 can be found in Figure 6 . Note that in this example the state estimation techniques without state equality constrains were used. Therefore, the predicted occupancy could be greater than $100 \%$.

\begin{tabular}{|c|c|c|c|c|c|}
\hline & $\frac{1}{n_{q}} \sum_{i} J_{I_{i}, t=1}^{P}$ & $\frac{1}{n_{q}} \sum_{i} J_{O_{i}}^{P}, t=1$ & $\frac{1}{n_{q}} \sum_{i} J_{Y_{i}, t=1}^{P}$ & $\frac{1}{n_{q}} \sum_{i} J_{I_{i}^{S L}, t=1}^{P}$ & $J^{S}$ \\
\hline$J$ (all arms) & 2.3468 & 33.4146 & 4.4227 & 2.7443 & 9.2226 \\
\hline $\begin{array}{c}J \text { (arms } 2,3,4, \\
5,6 \text { and } 8 \text { only) }\end{array}$ & 2.0566 & 11.7842 & - & 2.2878 & 4.6485 \\
\hline $\begin{array}{c}\text { Max. value of } \\
y_{i} \text { or } x_{i}\end{array}$ & 26 & 92.6427 & 26 & 25 & 61 \\
\hline
\end{tabular}

Table 2. The values of criterion (10) for the input intensities $I_{1}-I_{6}$, output occupancies $O_{1}-O_{6}$, output intensities $Y_{1}-Y_{8}$ and the intensities on the stop-lines $I_{1}^{S L}-I_{6}^{S L}$ and criterion (11) for the queue lengths. The last row contains maximal values of the corresponding quantities in the states or measurements.

On the other hand, in the standard (not oversaturated) situation, the measured quantities by the detector represent the number of arrived vehicles quite well and thus the quality of the estimated queue length and the predicted intensity and occupancy is significantly better, see Figure 7, where the traffic flow on arm 3 is illustrated.

For completeness Table 2 presents the validation results for this micro-region. Contrary to the previous example the criteria are evaluated over quantities for all arms and for all arms with standard, i.e. not oversaturated, traffic flows. The last table row presents again the maximal values of the corresponding quantities.

\section{Conclusion}

This chapter deals with the model design of traffic systems. The main aims were to introduce the modelling technique and to present validation methods for the designed models.

The proposed modelling technique is based on the traffic flow conservation principle. The use of the technique was illustrated on three simple common micro-regions that can constitute base for larger and more complex micro-regions. For the purposes of the model validation two criteria based on estimated state and predicted system output were proposed. Two designed models were validated using these two criteria on base of true and synthetic data.

The illustrating examples showed that the proposed models represent a sufficiently accurate description of the traffic systems.

The future work will be geared toward simpler and more straightforward description of the occupancies and toward the use of the proposed modelling technique for purposes of traffic control system. 


\section{Acknowledgement}

This work was supported by the Ministry of Education, Youth and Sport of the Czech Republic, project No. 1M0572.

\section{References}

Aboudolas, K.; Papageorgiou, M. \& Kosmatopoulos, E.(2007). A quadratic programming approach for the signal control problem in large-scale congested road networks. Proceedings of the Sixth Triennial Symposium on Transportation Analysis, pp. 1-6, Phuket Island, Thailand, June 2007.

Ackerman, N. A. (2000). Highway capacity manual. Transportation research board, Washington. D.C., USA.

AIMSUN: Users manual, (2004). Transport Simulation Systems. http://www.aimsun.com/, 2004.

Chilukuri, B. R.; Perrin, J. \& Martin, P. T. (2004). SCOOT and incidents - performance evaluation in simulated environment. Freeway Operations and Traffic Signal Systems, Vol. 1867, 2004, pp. 224-232, ISSN: 0361-1981

Courage, K. and Wallace, C.E. (1991). TRANSYT-7F User's Guide, Federal Highway Administration, Washington, D.C.

Duník, J. \& Šimandl, M (2008). Estimation of state and measurement noise covariance matrices by multi-step prediciton. Accepted for the 17th IFAC World Congress, Seoul, Korea, July 2008.

Gazis, D. C. (1964). Optimum Control of a System of Oversaturated Source. Operations Research, Vol. 12, No. 6, Special Transportation Science Issue, pp. 815-831.

Green, D. H. (1967). Control of Oversaturated Intersections. Operation Research, Vol. 18, No. pp. 161-173.

Hegyi, A.; Girimonte, D.; Babuška, R. \& De Schutter, B. (2006). A comparsion of filter configurations for freewaytraffic state estimation. In Proceedings of the 2006 IEEE Intelligent Transportation Systems Conference (ITSC 2006), pp. 1029-1034, Toronto, Canada, September 2006, ISBN: 1-4244-0094-5

Homolová, J. \& Nagy, I. (2005). Traffic model of a microregion. Preprints of the 16th IFAC World Congress, pp. 1-6, Prague, Czech Republic, July 2005, ISBN: 0-08-045108-X

Hunt, P. B.; Robertson, D. I.; Bretherton, R. D. and Royle, M. C. (1982). The SCOOT on-line traffic signal optimization technique. Traffic Eng. Control. Vol.23, pp 190-192.

Julier, S. J. \& Uhlmann, J. K. Unscented filtering and nonlinear estimation (2004). Proceedings of IEEE, Vol. 92, No. 3, Dec 2004, pp. 401-421, ISSN 0018-9219

Kara, K. \& Shabin, R. (2000). Effect of vehicle type on the capacity of signalized intersection: the case of light-duty trucks, Journal of Transportation Engineering, vol. 126, no. 6, pp. 506-512

Kosmatopoulos, E. and et al. (2006) International comparative field evaluation of a trafficresponsive signal control strategy in three cities. Transportation research part A policy and practice, Vol. 40, No. 5, pp. 399-413, 2006. ISSN 0965-8564

Kratochvílová, J. \& Nagy, I. (2004). Traffic control of microregion. CMP'04: Multiple Participant Decision Making, Theory, algorithms, software and applications, ed. 
Andrýsek, J. et al., pp. 161-171, Adelaide, May 2004. Advanced Knowledge International.

Ljung, L. (1999). System identification: theory for the user. UpperSaddle River, ISBN: 0-13656695-2, NJ: Prentice-Hall.

Lowrie, P. R. (1982). SCATS: The Sydney co-ordinated adaptive traffic system - Principles, methodology, algorithms. In Proceedings of IEE Int. Conf. Road Traffic Signalling. London. England. Pp. 67-70.

Mehra, R.K. (1972). Approaches to adaptive filtering. IEEE Transactions on Automatic Control, Vol. 17, No. 10, pp. 693-698, ISSN: 0018-9286

Mihaylova, L.; Boel, R. \& Hegyi, A. (2006). Freeway traffic estimation within particle filltering framework. Automatica, Vol. 43, No. 2, pp. 290-300, ISSN: 0005-1098

Moor, J.E.; Mattingly, S.P.; MacCArley, C.A. \& McNally, M.G. (2005). Anaheim advanced traffc control system filed operation test: A technical evaluation of SCOOT. Transportation Planning and Technology, Vol. 28, No. 6, pp. 465-482, ISSN: 03081060

Nørgaard, M.; Poulsen, N. K. \& Ravn, O. (2000). New developments in state estimation for nonlinear systems. Automatica, vol. 36, No. 11, pp. 1627-1638, ISSN: 0005-1098

Papageorgiou, M.; Diakaki, C.; Dinopoulou, V.; Kotsialos, A. \& Wang, Y.. Review of road traffic control strategies. Proceedings of IEEE, Vol. 91, No. 12, 2003, pp. 2043-2067, ISSN 0018-9219

Pearson, R. (2000). Traffic signal control. ITS Decision Report

Pecherková, P. \& Duník, J. (2006). Using derivative-free methods for estimation of intersection queue lengths. In Proceedings of the 11th International Conference of Hong Kong Society for Transportation Studies, Sustainable Transportation, pp. 739, Hong Kong, December 2006. Hong Kong Society for Transportation Studies.

Pecherková, P.; Homolová, J. \& Duník, J.(2007). Estimation of state and parameters of traffic system. Proceedings of the Fourth International Conference on Informatics in Control, Automation and Robotics, pp. 223-228, Angers, France, May 2007.

Robertson, D. I. (1969). Transyt: a traffic network study tool. Technical report, 1969.

Simon, D. \& Chia, T. L.. Kalman filtering state equality constrains. IEEE Transactions on Aerospace and Electronic Systems, vol. 38, No. 1, pp. 128-136, ISSN: 0018-9251

Simon, D. \& Simon, D. L. (2003). Aircraft turbofan engine health estimation using constrained Kalman filtering. ASME Turbo Expo 2003, pp. 1-8, Atlanta, GA, USA, June 2003.

Tichý, T. (2007). Evaluation methodology for traffic control system. Preprints of the ITS Prague '07 (in Czech), Prague, Czech republic, May 2007, ISBN: 978-80-239-93111-0

Verdú, S. \& Poor, H.V. (1984). Minimax linear observers and regulators for stochastic systems with uncertain second-order statistics. IEEE Transactions On Automatic Control, vol. 29, No. 6, pp. 499-511, ISSN: 0018-9286

Šimandl, M. \& Duník, J. (2006). Design of derivative-free smoothers and predictors. Preprints of the 14th IFAC Symposium on System Identification, pp. 991-996, Newcastle, Australia, March 2006. 
Wan, E. A.; van der Merwe, R. \& Nelson, A. T. (2000). Dual estimation and the unscented transformation. Advances in Neural Information Processing Systems 12, ed. Solla, S. A. et al., pp. 666-672, MIT Press, ISBN: 0-262-19450-3

Wong, S. C., Wong, W. T.; Xu, J. \& Tong, C. O. A time-dependent TRANSYT traffic model for area traffic control. In Traffic and Transportation Studies 2000, Beijing, China. 